\title{
Quality of Life and Osteoporotic Alterations in Patients with and without Low Bone Mineral Density: A Retrospective Study
}

Christyan Hiroshi Iida ${ }^{1}$, Natália Isis Caires Lavor ${ }^{1}$, Luciana Munhoz ${ }^{1}$, Reinaldo Abdala Júnior ${ }^{1}$, Rogério Abdala ${ }^{2}$ and Emiko Saito Arita ${ }^{1}$

1. Department of Stomatology, School of Dentistry, University of São Paulo, 2227 Lineu Prestes Avenue, São Paulo 05508-000, SP, Brazil

2. CDB (Centro de Diagnósticos Brasil), Rua Marselhesa, 500-Vila Mariana, São Paulo SP, Brazil

\begin{abstract}
Purpose: The objectives of this investigation were to compare the World Health Organization abbreviated instrument for quality of life assessment (WHOQOL-bref) scores of osteoporotic and non-osteoporotic Brazilian patients and to evaluate the correlation between mandibular cortical index (MCI) and bone densitometry (DXA) results in both groups. Methods: This research was conducted with an initial sample of 74 female participants who underwent panoramic radiography and forearm densitometry. Participants were divided into two groups: osteoporotic and non-osteoporotic patients. A quality of life assessment questionnaire was administered. The scores from the questionnaire were compared between the two groups and the correlation between DXA and MCI was analyzed. Results: Psychological and social domains in osteoporotic patients presented lower scores (12.65 and 6.00 respectively) when compared to non-osteoporotic patients (14.70 and 13.97 respectively). MCI was inversely correlated with DXA. Conclusions: According to WHOQOL-bref, osteoporotic patients may have lower quality of life scores when considering psychological and social relationships. MCI is inversely correlated with T-scores but demonstrates greater correlation when considering non-osteoporotic patients with higher T-score values.
\end{abstract}

Key words: Osteoporosis, panoramic radiography, bone loss, quality of life, bone mineral density.

\section{Introduction}

Osteoporosis is defined as systemic disease that affects bone metabolism. It is characterized by the decrease of bone mineral density (BMD) with bone tissue altered microarchitecture, resulting in increased bone fragility and fracture risk [1]. Osteoporosis bone loss is usually painless, and symptoms will emerge only when a fragility fracture occurs [2]. Osteoporosis morbidity is essentially caused by hip, vertebrae, and radius fractures [3]. Impaired quality of life (QL) secondary to fragility fractures is frequently observed in osteoporotic patients [4].

Corresponding author: Luciana Munhoz, DDS, MS, research fields: bone quality assessment, oral diagnosis, and imaging.
QL is negatively affected by osteoporosis as well as decreased BMD and fragility fractures [5]. Decreased QL in osteoporotic patients is predominantly caused by spine or hip fractures, which lead to pain as well as physical and social function impairment [3]. QL is valuable for evaluating the effectiveness of osteoporosis treatment and patients' perspectives [6].

To evaluate the QL of a variety of diseases, QL questionnaires were developed; one of these is the "World Health Organization abbreviated instrument for quality of life assessment” (WHOQOL-bref), which was validated for 30 languages. WHOQOL-bref has 24 questions comprising four domains: physical, psychological, social relations, and environment [7].

The early reduction of BMD can be detected by dual-energy X-ray absorptiometry (DXA), which is 
considered the gold standard for identifying osteoporosis due to its high accuracy and precision in detecting low bone mass [8].

However, the high cost and the scarce availability of DXA equipment may be a problem in emerging countries and access to the examination is burdensome. Therefore, alternative screening tools to detect patients at osteoporosis risk have been developed, such as radiomorphometric indexes on panoramic radiographs. Panoramic radiography relies on a low cost, low dose of radiation. It is widely used in dental practice and offers excellent visibility of facial anatomical structures. Several studies have applied this examination in assessing mandibular low BMD by analyzing the shape and thickness of the mandibular cortex [9-11]. One of the most established techniques is the Klemetti index [12], also known as Mandibular Cortical Index (MCI). MCI is a qualitative assessment of the appearance of the inferior cortex of the mandible on panoramic radiographs.

Thus, the objectives of this investigation are:

(1) To compare WHOQOL-bref scores in osteoporotic and non-osteoporotic Brazilian patients;

(2) To evaluate the MCI correlation with DXA results in both groups.

\section{Materials and Methods}

\subsection{Study Participants, Inclusion and Exclusion Criteria, and Data Collection}

This research was conducted with 79 participants referred for dental treatment at the Dentistry School between 2010 and 2014 who had undergone panoramic radiographic examination (at the beginning of dental treatment) and forearm DXA (for screening osteoporosis). All patients willing to participate in this investigation signed an informed consent form, and approval was obtained from the Ethics Committee of the university (number FR358902; protocol 145/10). The guidelines of Helsinki were followed in this investigation.
Among the initial sample of 79 female participants, 5 participants who did not undergo panoramic radiographic examination and forearm DXA on the same day or who had technical failures were excluded. Participants were divided into two groups (normal and low BMD) according to DXA results; BMD was defined as "low" if T-score was smaller than-2.5 SD [13].

The participants completed a form with personal information (e.g., gender and age), medical history (e.g., menopause, medications, comorbidities, and previous fractures). A previously validated life-quality questionnaire was applied (WHOQOL-bref, Brazilian Portuguese version) [14]. WHOQOL-bref consists of a total of 26 questions. Twenty-four questions are grouped in four main subjects: physical health, psychological health, social relationships, and environment [14]. The overall quality of life score is determined by the following two questions: "How would you rate your quality of life?" and "How satisfied are you with your health?” $[15,16]$. The score of each domain varies from zero to 20 , in which zero is considered the worst and 20 the best quality of life [16]; however, for the first 2 questions, the maximum score is 25 points [15]. When the quality of life questionnaire was applied, patients were instructed by researchers about how to fill the form; when asked for help, the researcher was limited to re-reading the questions only.

\subsection{Dual X-Ray Absorptiometry}

Forearm (proximal radius) bone densitometry measurements were conducted with peripheral dual-energy X-ray absorptiometry (pDEXA, Norland, Norland Medical Systems, Inc., White Plains, NY, USA). The scanning resolution was $1.00 \mathrm{~mm} \times$ $1.00 \mathrm{~mm}$

\subsection{Panoramic Radiographs and MCI Assessment}

All digital panoramic radiographic images were taken using the same device (Kodak 8000, Eastman 
Kodak Company, Rochester, USA). All images were processed on the same software (ImageJ, National Institutes of Health, Bethesda, MD, USA).

The MCI was assessed by evaluating the appearance of the cortical bone at the mandibular endosteal margin on both sides of the mandible, from an anterior limit below the mental foramen to the posterior limit at the mandibular angle, using a previously described classification [10, 12]. The mandibular endosteal margin was classified as follows: $\mathrm{C} 1$ = normal, when presenting sharp and marked continuous endosteal margin; C2 = moderately eroded, when presenting evidence of lacunar resorption or linear cortical residues; and C3 = severely eroded, when evident porosity was noticed or when presenting persistent and abundant linear bone gaps, reduced thickness, and loss of sharpness of the mandibular endosteal margin.

All panoramic radiomorphometric evaluation was performed independently in a random order by three trained observers. Intraobserver reliability was analyzed between measurements performed one week apart to eliminate memory bias.

\subsection{Statistical Analysis}

The WHOQOL-bref scores were transformed and presented according to the WHOQOL-bref scoring instructions [14].

All statistical analyses were performed using the IBM SPSS Statistics software (SPSS, Inc., Chicago, IL, USA), version 24.0, considering a significance level of $p<0.05$. Normality was assessed for variables using the Shapiro-Wilk test. Statistical analysis was divided into different stages:

The WHOQOL-bref domain scores from normal and low BMD group were compared, using $t$-test or Mann-Whitney test.

Correlation between T-scores and MCI of each group was determined, using the Spearman correlation test.

Intra- and interobserver agreements were evaluated using the Kappa test for MCI.

\section{Results and Discussion}

\subsection{Descriptive Data}

A total of 74 patients, divided into 2 groups according to BMD (normal: 37 patients; low BMD: 37 patients) were analyzed. Sample demographic characteristics according to the different groups are described in Table 1.

\subsection{WHOQOL-Bref Domain Scores}

Median scores of each domain and differences between groups are shown in Table 2. Although no differences between physical health scores were found, psychological health and social relationships scores were lower in the low-BMD patient group as compared to the normal (non-low) BMD group; however, regarding environment, the low BMD group presented higher scores. Questions 1 and 2, which consider the overall QL, exhibited no score differences between the two groups evaluated.

\subsection{Correlation between T-Scores and MCI of Each Group; Intra- and Interobserver Agreement Results}

Median T-scores and the correlation between MCI and T-scores from the two groups are shown in Table 3. A moderate correlation was found in the normal BMD group; a weak correlation was found in the low BMD group.

Intraobserver agreement according to the Kappa test was 0.78 ; interobserver agreement was 0.89 .

\subsection{Results Discussion}

Osteoporosis is one of the most common osteometabolic diseases worldwide and is the primary cause of fragility fractures [17]. In this study, the quality of life aspects of osteoporotic patients was compared to those of non-osteoporotic patients by using a QL questionnaire, as well as by the correlation between the T-scores obtained by peripheral DXA and panoramic radiographs. The instrument applied to 
Table 1 Sample characteristics according to: schooling, ethnicity, age, gender, diseases that affects de bone metabolism and previous fracture history.

\begin{tabular}{|c|c|c|c|}
\hline Category & Characteristic & $\begin{array}{l}\text { Number of participants } \\
\text { Normal BMD group }\end{array}$ & $\begin{array}{l}\text { Number of participants } \\
\text { Low BMD group }\end{array}$ \\
\hline \multirow[t]{3}{*}{ Schooling } & Graded School & 14 & 17 \\
\hline & High School & 10 & 09 \\
\hline & College & 13 & 11 \\
\hline \multirow[t]{3}{*}{ Ethnicity } & Caucasian & 27 & 26 \\
\hline & Afrodescendants & 01 & 02 \\
\hline & Asian & 09 & 09 \\
\hline \multirow[t]{4}{*}{ Age } & $40-50$ years & 05 & 05 \\
\hline & $51-60$ years & 05 & 02 \\
\hline & $61-70$ years & 14 & 16 \\
\hline & More than 71 years & 13 & 14 \\
\hline \multirow[t]{2}{*}{ Gender } & Female & 27 & 33 \\
\hline & Male & 10 & 04 \\
\hline \multirow[t]{4}{*}{ Diseases that affects bone metabolism } & Diabetes & 04 & 03 \\
\hline & Thyroid disease & 03 & 04 \\
\hline & Other & 04 & 06 \\
\hline & None & 26 & 24 \\
\hline \multirow[t]{2}{*}{ Previous history of fracture } & Spine & 0 & 01 \\
\hline & Leg or foot & 02 & 02 \\
\hline No previous history of fracture & & 35 & 34 \\
\hline Total of participants & & 37 & 37 \\
\hline
\end{tabular}

Table 2 Median scores of each group, according to each domain.

\begin{tabular}{lllllll}
\hline Group & $\begin{array}{l}\text { Physical health } \\
\text { (domain 1) }\end{array}$ & $\begin{array}{l}\text { Psychological health } \\
\text { (domain 2) }\end{array}$ & $\begin{array}{l}\text { Social relationships } \\
\text { (domain 3) }\end{array}$ & $\begin{array}{l}\text { Environment } \\
\text { (domain 4) }\end{array}$ & Question 1 & Question 2 \\
\hline Normal BMD & 15.41 (IR4) & 14.70 (IR3)* & $13.97($ IR4)* & $13.00($ IR3)* & 18.92 (IR5) & 17.03 (IR5) \\
Low BMD & 15.41 (IR4) & 12.65 (IR8)* & $06.00($ IR2)* & $15.16($ IR4)* & 18.78 (IR5) & 17.97 (IR5) \\
\hline
\end{tabular}

Abbreviations: IR: interquartile range.

* indicates difference between groups considering a significance.

Table 3 Median T-scores and correlation test results.

\begin{tabular}{lll}
\hline Group & T-score & Correlation MCI X T-score* \\
\hline Normal BMD & -1.57 & -0.411 \\
Low BMD & -3.83 & -0.266 \\
\hline
\end{tabular}

* According to Spearman correlation test.

assess the QL was the WHOQOL-bref questionnaire, an instrument that shows good performance and reliability and is validated to the Brazilian population [16].

The results of this study suggest that non-osteoporotic patients have higher scores in some QL assessments, particularly regarding psychological health (domain 2) and social relationships (domain 3). These results are in concordance with previous studies, which mentioned that osteoporosis can lead to socially and psychologically unfavorable alterations in patients with the disease [18].

Regarding the lower scores in the psychological domain, it is well known that there is an association between osteoporosis and depression [19]. Low physical functioning related to low BMD and role limitations may lead to poor emotional status [20]. In postmenopausal women, who comprised the majority 
of participants in the low BMD group of the present study, the relationship between depression and osteoporosis is not clear. It is not well known if osteoporosis is a causal factor of depression or if depression is an outcome of osteoporosis [21].

In the present study, a greater difference between osteoporotic patients and non-osteoporotic patients was observed in the social relation domain. A patient with osteoporosis, with low BMD and higher fracture risk, may have a decrease in mobility, muscular strength, balance, and physical activity [22], which will certainly have a great impact on his or her behavior and societal interactions. These may affect both individual and family social roles, resulting in social non-reciprocity and social deprivation or isolation [18].

Low scores regarding physical health in osteoporotic patients were expected but, surprisingly, no differences between osteoporotic and normal patients' scores were found. Statistically significant differences were found in questions 1 and 2, which may be related to the fact that there is controversy about whether osteoporosis is a painful disease [2, 23]. Pain occurs essentially when a low-energy fracture occurs [2]. Although osteoporosis is often a painless disease, with pain usually related to major fragility fractures, patients may present chronic back pain due to microfractures in vertebrae caused by mechanical pressure, which leads to compression of spinal nerves [24]. In the present study, only a few patients had experienced previous fractures. One of the questions evaluated in the physical domain (domain 1) was "In what measure do you think your physical pain impedes you from doing what you need?” In both groups, the most common answer chosen was "nothing."

Regarding the environment (domain 4), in which domain scores were higher for osteoporotic patients than for non-osteoporotic, a different result was expected, mainly when considering the quality of and access to health services in Brazil, which is influenced by demographic and socioeconomic factors [25] including inequalities in the utilization of health services. The scores found in this domain may not be associated with the presence or absence of the osteoporosis itself, but with the locality in which the patient lives, and factors such as security, financial resources, or transportation.

Some self-assessment questions about patients' quality of life, personal satisfaction, and others, had predominantly favorable answers for both osteoporotic and non-osteoporotic patients. This similarity may be related to the fact that most of the osteoporotic patients already knew that they had the disease.

When considering the correlation between MCI and T-score, it was noticed that there is an inverse negative correlation, which showed that the higher the MCI classification, the lower the T-score. Notwithstanding, a greater correlation was observed among individuals with normal BMD when compared to those with low BMD. This dissimilarity may be because C1 classifications, frequently related to normal BMD patients, are much easier to identify than C2 or C3 classifications.

The limitations of the present study were the small number of patients and its retrospective design. Further larger sample studies should be performed to confirm the results. Some domains had somewhat unexpected differences or similarities. After all, the concept of quality of life is tied to the subjectivity of feelings, such as happiness or pleasure, inherent to the individual itself, and economic and social expectations within the environmental media whose individual is inserted.

\section{Conclusion}

In conclusion, according to WHOQOL-bref, osteoporotic patients may have lower QL scores when considering psychological and social relationships. Low-BMD patients had higher QL assessment scores than non-osteoporotic patients, but these results may 
be associated with the locality in which the patient lives and factors such as security, financial resources, or transportation. MCI is inversely correlated with T-scores, but demonstrates greater correlation when considering non-osteoporotic patients with higher T-scores values.

\section{Acknowledgments}

We would like to thank the CDB (Centro de Diagnósticos Brasil) and Doctor Rogério Abdala for providing us with the scientific material required to perform this study.

\section{Conflicts of Interest}

Christyan Hiroshi Iida, Natalia Isis Caires Lavor Luciana Munhoz, Rogério Abdala, Reinaldo Abdala Júnior and Emiko Saito Arita declare no conflicts of interest.

\section{Funding}

This study was financed in part by the "Coordenação de Aperfeiçoamento de Pessoal de Nível Superior, Brasil (CAPES)—Finance Code 001”.

\section{References}

[1] WHO Study Group. 1994. “Assessment of Fracture Risk and Its Application to Screening for Postmenopausal Osteoporosis.” Report of a World Health Organ Tech Rep Ser. 843: 1-129.

[2] Vellucci, R., Terenzi, R., Kanis, J. A., Kress, H. G., Mediati, R. D., Reginster, J. Y., et al. 2018. "Understanding Osteoporotic Pain and Its Pharmacological Treatment.” Osteoporos Int. 29 (7): 1477-91.

[3] Lips, P., and Van Schoor, N. M. 2005. "Quality of Life in Patients with Osteoporosis.” Osteoporos Int. 16 (5): 447-55.

[4] Hutson, J. M., and Beasley, S. W. 1988. "Omphalocele, Cryptorchidism and Brain Malformations.” J Pediatr Surg. 23 (5): 506-7.

[5] Orimo, H., Nakamura, T., Hosoi, T., Iki, M., Uenishi, K., Endo, N., et al. 2012. “Japanese 2011 Guidelines for Prevention and Treatment of Osteoporosis-Executive Summary.” Arch Osteoporos 7: 3-20.

[6] Urushihara, H., Yoh, K., Hamaya, E., Taketsuna, M., and Tanaka, K. 2014. "Responsiveness of the Japanese
Osteoporosis Quality of Life Questionnaire in Women with Postmenopausal Osteoporosis." Health Qual Life Outcomes 12: 178.

[7] Tavares, D. M., Matias, T. G., Ferreira, P. C., Pegorari, M. S., Nascimento, J. S., and Paiva, M. M. 2016. "Quality of Life and Self-esteem among the Elderly in the Community." Cien Saude Colet. 21 (11): 3557-64.

[8] Pallagatti, S., Parnami, P., Sheikh, S., and Gupta, D. 2017. "Efficacy of Panoramic Radiography in the Detection of Osteoporosis in Post-Menopausal Women When Compared to Dual Energy X-Ray Absorptiometry.” Open Dent J. 11: 350-9.

[9] Bajoria, A. A., Kamath, G., Babshet, M., Patil, P., and Sukhija, P. 2015. "Evaluation of Radiomorphometric Indices in Panoramic Radiograph-A Screening Tool.” Open Dent J. 9: 303-10.

[10] Munhoz, L., Aoki, E. M., Cortes, A. R. G., de Freitas, C. F., and Arita, E. S. 2018. "Osteoporotic Alterations in a Group of Different Ethnicity Brazilian Postmenopausal Women: An Observational Study.” Gerodontology.

[11] Munhoz, L., Cortes, A. R., and Arita, E. S. “Assessment of Osteoporotic Alterations in Type 2 Diabetes: A Retrospective Study.” Dentomaxillofac Radiol. 46 (6): 20160414.

[12] Klemetti, E., Kolmakov, S., and Kröger, H. 1994. "Pantomography in Assessment of the Osteoporosis Risk Group.” Scand J Dent Res. 102 (1): 68-72.

[13] Kanis, J. A. 1994. “Assessment of Fracture Risk and Its Application to Screening for Postmenopausal Osteoporosis: Synopsis of a WHO Report.” WHO Study Group. Osteoporos Int. 4 (6): 368-81.

[14] WHOQOL Group. 1998. "Development of the World Health Organization WHOQOL-BREF Quality of Life Assessment.” The Psychol Med. 28 (3): 551-8.

[15] Castro, P. C., Driusso, P., and Oishi, J. 2014. "Convergent Validity between SF-36 and WHOQOL-BREF in Older Adults.” Rev Saude Publica. 48 (1): 63-7.

[16] Fleck, M. P., Louzada, S., Xavier, M., Chachamovich, E., Vieira, G., Santos, L., et al. 2000. "Application of the Portuguese Version of the Abbreviated Instrument of Quality Life WHOQOL-Bref.” Rev Saude Publica 34 (2): 178-83.

[17] Catalano, A., Martino, G., Morabito, N., Scarcella, C., Gaudio, A., Basile, G., et al. 2017. "Pain in Osteoporosis: From Pathophysiology to Therapeutic Approach.” Drugs Aging 34 (10): 755-65.

[18] Lasaite, L., and Krasauskiene, A. 2009. "Psychological State, Quality of Life, and Body Composition in Postmenopausal Women with Osteoporosis in Lithuania." Arch Osteoporos 4 (1-2): 85-90.

[19] Mollard, E., Bilek, L., and Waltman, N. 2018. “Emerging 
Evidence on the Link between Depressive Symptoms and Bone Loss in Postmenopausal Women.” Int J Women Health 10: 1-9.

[20] Tashiro, A., Kakuta, H., Tanaka, N., and Takeuchi, Y. 2006. "Relationship between Health-Related Quality of Life Domains and Bone Status in Postmenopausal Japanese Women.” Menopause 13 (5): 846-9.

[21] Gold, D. T., and Solimeo, S. 2006. "Osteoporosis and Depression: A Historical Perspective.” Curr Osteoporos Rep. 4 (4): 134-9.

[22] Dewan, N., MacDermid, J. C., MacIntyre, N. J., and Grewal, R. 2018. “Therapist's Practice Patterns for Subsequent Fall/Osteoporotic Fracture Prevention for Patients with a Distal Radius Fracture.” J Hand Ther.
[23] Lee, K. M., Chung, C. Y., Kwon, S. S., Kim, T. G., Lee, I. H., Jung, K. J., et al. 2015. "Bone Mineral Density Is Not Associated with Musculoskeletal Pain in Postmenopausal Korean Women Aged $\geq 50$ Years.” Clin Rheumatol. 34 (2): 337-43.

[24] Suzuki, M., Orita, S., Miyagi, M., Ishikawa, T., Kamoda, H., Eguchi, Y., et al. 2013. "Vertebral Compression Exacerbates Osteoporotic Pain in an Ovariectomy-Induced Osteoporosis Rat Model.” Spine 38 (24): 2085-91.

[25] Ascef, B. O., Haddad, J. P. A., Álvares, J., Guerra, A. A., Costa, E. A., Acurcio, F. A., et al. 2017. "Health-Related Quality of Life of Patients of Brazilian Primary Health Care.” Rev Saude Publica 51 (Suppl. 2): 22s. 